\title{
Low-dose Btk inhibitors selectively block platelet activation by CLEC-2
}

Haematologica 2021

Volume 106(1):208-219

\section{Correspondence:}

PHILLIP LR NICOLSON

p.nicolson@bham.ac.uk

\section{STEVE P WATSON}

s.p.watson@bham.ac.uk

CRAIG E HUGHES

c.e.hughes@reading.ac.uk

Received: February 8, 2019.

Accepted: January 15, 2020.

Pre-published: January 16, 2020.

https://doi.org/10.3324/haematol.2019.218545

(C)2021 Ferrata Storti Foundation

Material published in Haematologica is covered by copyright. All rights are reserved to the Ferrata Storti Foundation. Use of published material is allowed under the following terms and conditions:

https://creativecommons.org/licenses/by-nc/4.0/legalcode. Copies of published material are allowed for personal or internal use. Sharing published material for non-commercial purposes is subject to the following conditions:

https://creativecommons.org/licenses/by-nc/4.0/legalcode, sect. 3. Reproducing and sharing published material for commercial purposes is not allowed without permission in writing from the publisher.

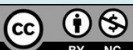

\section{Phillip L.R. Nicolson, ${ }^{1,2}$ Sophie H. Nock, ${ }^{3}$ Joshua Hinds, ${ }^{1}$ Lourdes Garcia-Quintanilla, ${ }^{1}$ Christopher W. Smith, ${ }^{1}$ Joana Campos, ${ }^{1}$ Alexander Brill,,${ }^{1,4}$ Jeremy A. Pike, ${ }^{1,5}$ Abdullah O. Khan, ${ }^{1}$ Natalie S. Poulter,${ }^{1,5}$ Deidre M. Kavanagh, ${ }^{1,5}$ Stephanie Watson, ${ }^{1}$ Callum N. Watson, ${ }^{1}$ Hayley Clifford, ${ }^{6}$ Aarnoud P. Huissoon, ${ }^{6}$ Alice Y. Pollitt, ${ }^{3}$ Johannes A. Eble, ${ }^{7}$ Guy Pratt, ${ }^{2}$ Steve P. Watson ${ }^{1,5}$ and Craig E. Hughes ${ }^{3}$}

${ }^{1}$ Institute of Cardiovascular Sciences, College of Medical and Dental Sciences, University of Birmingham, Birmingham, UK; ' ${ }^{2}$ Department of Haematology, Queen Elizabeth Hospital, Birmingham, UK; ${ }^{3}$ nstitute for Cardiovascular and Metabolic Research, Harborne Building, University of Reading, Reading, UK; ${ }^{4}$ Department of Pathophysiology, Sechenov First Moscow State Medical University (Sechenov University), Moscow, Russia; ${ }^{5}$ Centre of Membrane Proteins and Receptors (COMPARE), Universities of Birmingham and Nottingham, Midlands, UK; ${ }^{6}$ Department of Immunology, Heartlands Hospital, Birmingham, UK and ${ }^{7}$ Institute for Physiological Chemistry and Pathobiochemistry, University of Münster, Mübster, Germany

\section{ABSTRACT}

T nhibitors of Bruton tyrosine kinase (Btk) have been proposed as novel antiplatelet agents. In this study we show that low concentrations of the Btk inhibitor ibrutinib block CLEC-2-mediated activation and tyrosine phosphorylation including Syk and PLC $\gamma 2$ in human platelets. Activation is also blocked in patients with X-linked agammaglobulinemia (XLA) caused by a deficiency or absence of Btk. In contrast, the response to GPVI is delayed in the presence of low concentrations of ibrutinib or in patients with XLA, and tyrosine phosphorylation of Syk is preserved. A similar set of results is seen with the second-generation inhibitor, acalabrutinib. The differential effect of Btk inhibition in CLEC2 relative to GPVI signaling is explained by the positive feedback role involving Btk itself, as well as ADP-and thromboxane $\mathrm{A}_{2}$-mediated activation of $\mathrm{P} 2 \mathrm{Y}_{12}$ and $\mathrm{TP}$ receptors, respectively. This feedback role is not seen in mouse platelets and, consistent with this, CLEC-2-mediated activation is blocked by high but not by low concentrations of ibrutinib. Nevertheless, thrombosis was absent in eight out of 13 mice treated with ibrutinib. These results show that Btk inhibitors selectively block activation of human platelets by CLEC-2 relative to GPVI suggesting that they can be used at low doses in patients to target CLEC-2 in thrombo-inflammatory disease.

\section{Introduction}

The C-type lectin receptor CLEC-2 is expressed at high levels on platelets and at low levels on a subpopulation of hematopoietic cells. ${ }^{1}$ Its only known physiological ligand is podoplanin. ${ }^{2}$ Recent studies have demonstrated a critical role for CLEC-2 in inflammation-driven venous thrombosis, ${ }^{3,4}$ generating interest in drugs that target CLEC-2 in thrombo-inflammatory disorders while preserving hemostasis. This interest has been further fueled by the demonstration that CLEC-2 plays a minimal or negligible role in hemostasis. ${ }^{5}$

CLEC-2 has a cytoplasmic tail containing a single tyrosine in a conserved YxxL sequence in a motif that represents half of an immunoreceptor tyrosine-based activation motif (ITAM), known as a hemITAM. The conserved tyrosine is phosphorylated by the tyrosine kinase Syk. ${ }^{6}$ This leads to recruitment of Syk through binding of its tandem $\mathrm{SH} 2$ domains and initiation of a downstream signaling cascade involving Src, Syk and Tec (including Bruton tyrosine kinase [Btk]) kinases, various adapter proteins including LAT, Gads, Grb-2 and SLP-76, the Vav family 
GTP exchange proteins and the effector protein, PLC $\gamma 2$. ${ }^{9}$ This hemITAM signaling pathway is similar to that used by the YxxL-containing platelet ITAM receptors such as the collagen receptor GPVI-FcR $\gamma$ complex and the low affinity immune receptor Fc $\gamma$ RIIA ${ }^{10}$. A fundamental difference between these pathways is, however, that Syk binds to two phosphorylated tyrosines within a single ITAM in GPVI-FcR $\gamma$ and FcyRIIA, and to two phosphorylated hemITAM in separate cytosolic receptor chains of CLEC$2 .{ }^{11}$ Another difference between CLEC-2 and GPVI signaling in human platelets is the critical dependence of CLEC2 on positive feedback signaling through ADP and thromboxane $\mathrm{A}_{2}\left(\mathrm{TxA}_{2}\right)$, actin polymerization, and the small GTPase Rac. ${ }^{8}$ In contrast these positive feedback pathways play a relatively minor role in CLEC-2 signaling in mouse platelets. ${ }^{12}$

Recently, irreversible inhibitors of the Tec kinase Btk have entered clinical use for the treatment of B-cell malignancies. ${ }^{13,14}$ These include the first-generation inhibitor ibrutinib and the second-generation inhibitor acalabrutinib. Ibrutinib is associated with a significant increase in major bleeding which is much reduced with the secondgeneration inhibitor acalabrutinib. ${ }^{14,15}$ Patients with Xlinked agammaglobulinemia (XLA) who have functiondisrupting mutations or loss of Btk do not, however, have increased bleeding. This observation, coupled with protein biochemistry measurements, have led us to conclude that the bleeding induced by ibrutinib is due to the high dosing strategy and off-target effects. ${ }^{16}$ Platelets express two members of the Tec family of tyrosine kinases, namely Btk and Tec. Btk has an approximate 10- to 20fold greater level of expression in both human and mouse platelets. ${ }^{17,18}$ Various studies have shown redundancy between the two kinases downstream of GPVI, with Tec able to partially compensate for the absence of Btk in human platelets from patients with XLA and in mouse platelets, which are deficient in Btk. ${ }^{16,19,20}$

Recently, Btk inhibitors have been proposed to represent a new class of antithrombotic drug ${ }^{21}$ based on the observation that they delay or inhibit ex vivo platelet activation by $\mathrm{GPVI}^{16,22-24}$ and by immobilized atherosclerotic plaque at arterial rates of flow. ${ }^{25}$ In addition, Btk inhibitors block platelet activation by CLEC-2. However, based on studies using ibrutinib-treated human platelets, Manne et al. have proposed that Btk, in contrast to GPVI, lies upstream of Syk in the CLEC-2 signaling cascade. ${ }^{26}$

In the present study, we demonstrate that the results of Manne et al. are explained by the positive feedback role of $\mathrm{ADP}$ and $\mathrm{TxA}$, and that concentrations of ibrutinib that have little or no effect on the response to GPVI stimulation selectively block activation by CLEC-2. This observation, together with the pivotal role of CLEC-2 in thrombo-inflammation, suggests that inhibitors of Btk represent new antiplatelet agents for thrombo-inflammatory disorders with minimal effect on hemostasis.

\section{Methods}

\section{Platelet preparation}

Blood was taken from consenting patients or healthy, drugfree volunteers, into $4 \%$ sodium citrate as previously described. ${ }^{16}$ Mouse blood was drawn from mice asphyxiated with $\mathrm{CO}_{2}$ following isoflurane anesthesia by inferior vena cava (IVC) puncture and collected into acid-citrate-dextrose. Platelet-rich plasma was obtained by centrifugation. Washed human and mouse platelets were obtained by further centrifugation of the plateletrich plasma in the presence of prostacyclin and resuspended in modified Tyrode buffer as previously described. ${ }^{16}$ Platelets were used at a cell density of $4 \times 10^{8} / \mathrm{mL}$ for aggregometry and biochemical studies.

\section{Light transmission aggregometry}

Aggregation was monitored by light transmission using a Model 700 aggregometer (Chronolog, Havertown, PA, USA) as previously described. ${ }^{16}$

\section{Protein phosphorylation}

Eptifibatide-treated, washed platelets were stimulated in a Model 700 aggregometer in the presence of ibrutinib or vehicle as described previously. ${ }^{16}$ Activation was terminated after 300 seconds by addition of reducing sample buffer. This was followed by lysate separation by sodium dodecylsulfate polyacrylamide gel electrophoresis, electro-transfer and western blot as described previously. ${ }^{16}$

\section{Inferior vena cava stenosis assay}

All mouse experiments were performed using wild-type (WT) mice on a C57B1/6 genetic background under Home Office project licenses P0E98D513 and PC427E5DD. Mice were sourced from Charles River UK Ltd. (Margate, UK). For ex vivo platelet function assays and in vivo thrombosis assays 8-week old C57Bl/6 WT male mice were treated by intraperitoneal injection with a total dose of $140 \mathrm{mg} / \mathrm{kg}$ of ibrutinib or vehicle $(5 \%$ dimethylsulfoxide, $30 \%$ polyethylene glycol $300,5 \%$ Tween 20 , $60 \%$ deionized water) in divided doses once daily over $2-4$ days. Blood was taken at the stated times and platelet function analysis was performed as described above using flow cytometry.

The IVC stenosis model was performed as described by Payne et al. ${ }^{3}$ In brief, mice were anesthetized using isoflurane and then a laparotomy was performed. Side branches of the IVC were identified and tied off before the IVC itself was stenosed with a ligature and a 30-gauge spacer to maintain a small degree of vessel patency. The incision was then closed and mice were allowed to recover from surgery. Buprenorphine was used preand post-operatively for analgesia. Mice were then culled $48 \mathrm{~h}$ after the surgery and the IVC was examined for the presence and size of thrombus.

\section{Other methods}

Details of reagents and methods used for flow cytometry, flow adhesion, imaging, image analysis and cell transfection are detailed in the Online Supplementary Material.

\section{Statistical analysis}

All data are presented as mean \pm standard error of the mean with statistical significance taken as $P<0.05$ unless otherwise stated. Statistical analyses were performed using the MannWhitney test, Fisher exact test, Welch $t$-test or one or two-way analysis of variance (ANOVA) with corrections for multiple comparisons, as stated. Statistical analysis of the half maximal inhibitory concentration $\left(\mathrm{IC}_{\text {so }}\right.$ ) values was performed using the Welch $t$-test. All statistical analyses were performed using GraphPad Prism 7 (GraphPad Software Inc. La Jolla, CA, USA).

\section{Ethical approval}

Ethical approval for collecting blood from patients and healthy volunteers was granted by the National Research Ethics Service (10/H1206/58) and Birmingham University Internal 
Ethical Review (ERN_11-0175), respectively. Work on patients with XLA has ethical approval via the University of Birmingham Human Biomaterials Resource Centre (16-251 Amendment 3).

\section{Results}

CLEC-2-mediated human platelet aggregation is inhibited by low concentrations of Btk inhibitors

To examine the role of Btk downstream of platelet CLEC-2 in humans we took washed platelets from healthy donors and added increasing concentrations of ibrutinib before stimulating the platelets with the CLEC2 agonist rhodocytin. As shown in Figure 1A, B, ibrutinib completely inhibited CLEC-2-mediated aggregation at concentrations as low as $70 \mathrm{nM}$. Inhibition was marked after 5 min incubation with ibrutinib and only increased slightly with times up to 60 min (Figure 1A, B and Online Supplementary Figure S1). Strikingly, the $\mathrm{IC}_{50}$ for inhibition was over 20 -fold lower than that required to block GPVImediated platelet aggregation ${ }^{16}$ (Table 1). This selective inhibition of the response to CLEC-2 activation is consistent with the results of Manne et al. ${ }^{26}$

We further studied the effect of in vivo Btk inhibition on platelet-rich plasma obtained from patients with chronic lymphocytic leukemia (CLL) treated with ibrutinib or the more specific second-generation Btk inhibitor acalabrutinib. Unlike platelets from patients treated with a control chemotherapy regimen (fludarabine, cyclophosphamide and rituximab; FCR), platelets from Btk inhibitor-treated patients did not aggregate even at high levels of CLEC-2 stimulation (Figure 1C). We have previously shown that platelet-rich plasma from Btk inhibitor-treated patients does, however, aggregate in response to GPVI and G protein-coupled receptor (GPCR) stimulation. ${ }^{16}$

\section{Adhesion to podoplanin under venous flow conditions is abrogated by Btk inhibition}

Given the role of platelet CLEC-2 and podoplanin in the thrombo-inflammatory and venous thrombosis models used by Hitchcock et al. ${ }^{4}$ and Payne et al. ${ }^{3}$ we investigated whether inhibition of CLEC-2-mediated platelet function
A

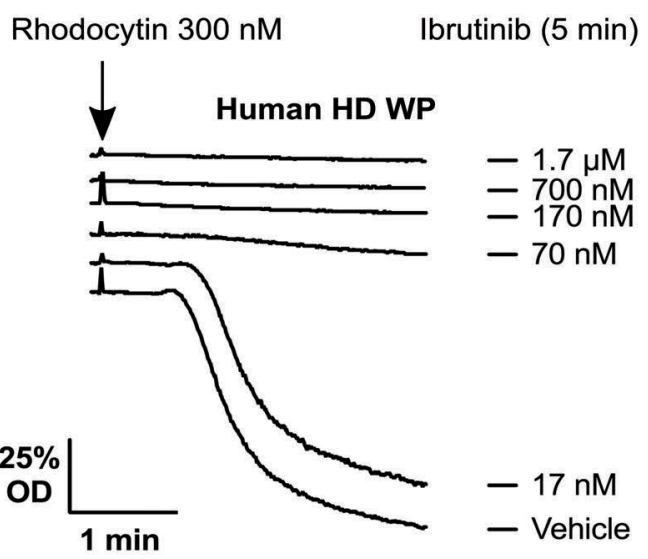

C i

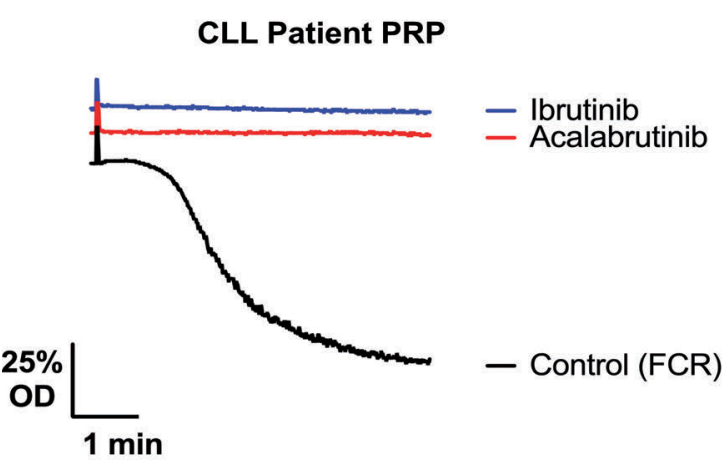

B

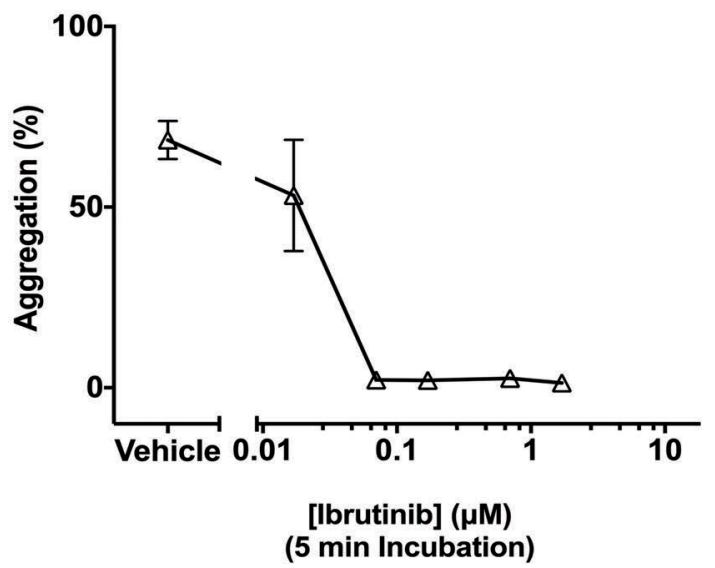

C ii

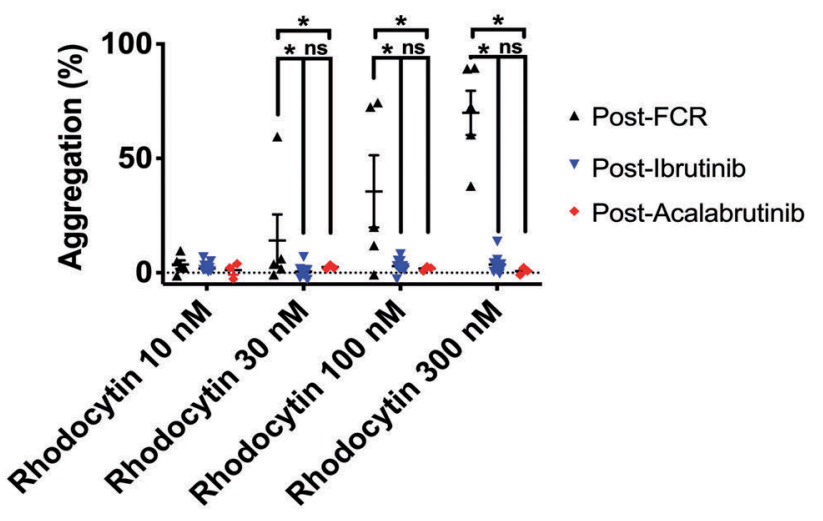

Figure 1. Btk inhibitors completely block CLEC-2-mediated platelet aggregation in vitro and ex vivo. Washed platelets at $4 \times 10^{8} / \mathrm{mL}$ isolated from healthy donors were stimulated with $300 \mathrm{nM}$ rhodocytin after incubation with ibrutinib or vehicle at the stated concentrations for 5 min. Light transmission aggregometry measurements were then undertaken for $5 \mathrm{~min}$. (A) Representative traces of three identical aggregation experiments. (B) Dose response curves ( $\mathrm{n}=3$ ). (C) Platlet-rich plasma was isolated from the blood of patients with chronic lymphocytic leukemia following treatment with fludarabine, cyclophosphamide and rituximab (FCR), ibrutinib or acalabrutinib based chemotherapy regimens. (i) Representative trace and (ii) mean \pm standard error of mean (SEM) (post-FCR, $n=5$; post-ibrutinib, $n=12$; post-acalabrutinib, $n=3$ ) of optical density 5 min after stimulation with rhodocytin $10-300 \mathrm{nM}$. All results are shown as mean \pm SEM. Statistical analysis was performed with a two-way analysis of variance with the Tukey correction for multiple comparisons. ${ }^{*} P<0.05$, ns=not significant. HD: healthy donor; WP: washed platelets; OD: optical density; CLL: chronic lymphocytic leukemia; PRP: platelet-rich plasma. 
by ibrutinib reduced or prevented adhesion to the endogenous ligand under flow. Blood from healthy donors, patients with XLA or those taking ibrutinib or acalabrutinib was flowed over podoplanin at a venous shear. Strikingly we found that both the adhesion and aggregate size of these platelets were markedly reduced when compared to those of platelets from healthy donor blood regardless of the method of Btk inhibition (Figure 2).

\section{Btk and Syk inhibition block CLEC-2-mediated Btk phosphorylation}

To examine the effect of ibrutinib on CLEC-2-mediated protein phosphorylation, ibrutinib-treated washed human platelets were lysed after stimulation with rhodocytin and lysates probed with phosphospecific antibodies. As shown in Figure 3A, phosphorylation of Syk Y525/6, SLP-76 Y145, LAT Y200, Btk Y223 and Y551, and PLC 2 Y1217 was blocked by 70 nM ibrutinib with IC values similar to those for aggregation (Table 1). This concentration of ibrutinib reduced total phosphotyrosine to basal levels and inhibited phosphorylation on all measured tyrosine sites except the constitutive phosphorylation of Src Y418. Loss of Src pY418 was seen at higher concentrations of ibrutinib (Figure 3Aiv) as in our previous study. ${ }^{16}$

We performed similar experiments with acalabrutinib and found that Btk Y223 phosphorylation was lost at a concentration $(3 \mu \mathrm{M})$ that also partially inhibited phosphorylation of Syk (Online Supplementary Figure S2). This concentration caused a marked reduction in platelet activation by CLEC-2 but no change in platelet aggregation in response to GPVI ligation, ${ }^{16}$ confirming the selectivity for

Table 1. Half maximal inhibitory concentration $\left(\mathrm{IC}_{\mathrm{s}}\right)$ values for all doseresponse curves shown in the figures in this publication.

\begin{tabular}{|c|c|c|c|}
\hline Figure & Dose-response curve & $\mathrm{IC}_{50}(\mu \mathrm{M})$ & $95 \%$ Cl \\
\hline 1B & HD Aggregation & 0.023 & $0.009-0.053$ \\
\hline 3Aii & HD Btk pY223 & 0.024 & $0.002-0.133$ \\
\hline 3Aii & HD PLC 2 pY1217 & 0.034 & $0.010-0.119$ \\
\hline 3Aiii & HD Syk pY525/6 & 0.052 & $0.020-0.150$ \\
\hline 3Aiii & HD LAT pY200 & 0.036 & $0.028-0.189$ \\
\hline 3Aiii & HD SLP76 pY145 & 0.032 & $0.015-0.066$ \\
\hline 3Aiii & HD Btk pY551 & 0.059 & $0.023-0.179$ \\
\hline 3Aiv & HD Src pY418 & - & - \\
\hline 3Bii & HD Aggregation (added $10 \mu \mathrm{M}$ ADP) & 0.034 & $0.017-0.07$ \\
\hline 3Biii & HD Btk pY223 (added $10 \mu \mathrm{M}$ ADP) & 0.006 & $0.003-0.009$ \\
\hline 3Biii & HD PLC 2 pY1217 (added $10 \mu \mathrm{M}$ ADP) & 0.023 & $0.014-0.037$ \\
\hline $6 \mathrm{~B}$ & WT Mouse WP Aggregation & 13.28 & $4.650-129.2$ \\
\hline 6Bii & WT Mouse Btk pY223 & 0.076 & $0.011-0.559$ \\
\hline 6Bii & WT Mouse PLC $\gamma 2$ Y753 & 0.074 & $0.026-0.200$ \\
\hline 6Bii & WT Mouse PLC 22 Y759 & 0.065 & $0.017-0.232$ \\
\hline 6Bii & WT Mouse PLC $\gamma 2$ Y1217 & 0.122 & $0.044-0.363$ \\
\hline 6Biii & WT Mouse Syk pY525/6 & - & - \\
\hline 6Biii & WT Mouse LAT pY132 & - & - \\
\hline 6Biii & WT Mouse LAT pY200 & - & - \\
\hline 6Biii & WT Mouse Btk pY551 & - & - \\
\hline 6Biv & WT Mouse Src pY418 & 0.35 & $0.126-1.036$ \\
\hline
\end{tabular}

Where no value is shown this indicates that the $\mathrm{IC}_{\text {so }}$ could not be calculated. $95 \%$ CI: $95 \%$ confidence interval; HD: healthy donor;WT: wild-type.
CLEC-2. The observation, however, of partial phosphorylation of Syk and complete abrogation of phosphorylation of Btk in the presence of acalabrutinib suggests that Btk does not lie upstream of Syk, in contrast to the conclusion of Manne et ll. $^{26}$

A further prediction in the model of Manne et al. ${ }^{26}$ in which Syk lies downstream of Btk, is that inhibition of Syk should not block phosphorylation of Btk. To test this, we incubated human platelets with increasing concentrations of the Syk inhibitor PRT 060318 prior to stimulation with rhodocytin. Lysates were then probed using phosphospecific antibodies to Syk pY525/6, LAT pY200, Btk pY551 and PLC $\gamma 2$ pY1217. Online Supplementary Figure S3 shows that phosphorylation of Syk, LAT, Btk and PLC $\gamma 2$ were lost in tandem, providing further evidence against the model. This result is in keeping with our previous results examining CLEC-2-mediated phosphorylation events in mice with a Syk mutation rendering it unable to undergo tyrosine phosphorylation. ${ }^{9}$

In summary, the above results show that low concentrations of ibrutinib and acalabrutinib selectively block platelet activation by CLEC-2 over GPVI and provide evidence against the model of Manne et al. in which Btk lies upstream of Syk.

\section{ADP rescues Syk phosphorylation in the presence of Btk inhibition}

We have previously shown that CLEC-2 signaling in human platelets is critically dependent on positive feedback signals from thromboxane TP and ADP P2Y receptors. ${ }^{8}$ Our standard platelet preparation method leads to partial desensitization of the ADP P2Y, receptor ${ }^{27}$ and loss of aggregation in response to $\mathrm{ADP}$ as a consequence of this (Online Supplementary Figure S4). This may have accentuated the previously described dependency on P2Y ${ }_{12}$ receptors. To investigate this, we repeated the studies on aggregation and protein phosphorylation but in the presence of excess ADP $(10 \mu \mathrm{M})$. Similar to the results with no added ADP, 70 nM ibrutinib inhibited aggregation (Figure 3Bii) and phosphorylation of Btk Y223 and PLCY2 Y1217 (Figure 3Bi-ii). In contrast, however, it did not inhibit phosphorylation of Syk Y525/6, LAT Y200 or Btk on its Src kinase phosphorylation site Y551 (Figure $3 \mathrm{Bi}$ and iii). The demonstration of phosphorylation of Syk, LAT and Btk on Y551 but absence of Btk autophosphorylation on Y223 in ibrutinib-treated human platelets confirms that Btk lies downstream of Syk in the CLEC-2 signaling cascade in human platelets.

\section{CLEC-2-mediated platelet aggregation and tyrosine phosphorylation are blocked in patients with Btk mutations}

Our previous study found off-target effects of ibrutinib even when used at concentrations as low as $70 \mathrm{nM} .{ }^{16}$ To investigate whether the potent inhibition of CLEC-2mediated platelet activation by ibrutinib was mediated by its blockade of Btk or an off-target effect, we studied platelet aggregation in response to rhodocytin in patients with XLA. CLEC-2-mediated aggregation and phosphorylation of PLC $\gamma 2$ Y1217 were blocked, whereas phosphorylation of Syk Y525/6 and LAT Y200 was only partially reduced (Figure 4). These findings demonstrate that the initial phosphorylation of Syk and LAT is independent of Btk and that this is then increased by a Btk-dependent pathway. 
A i

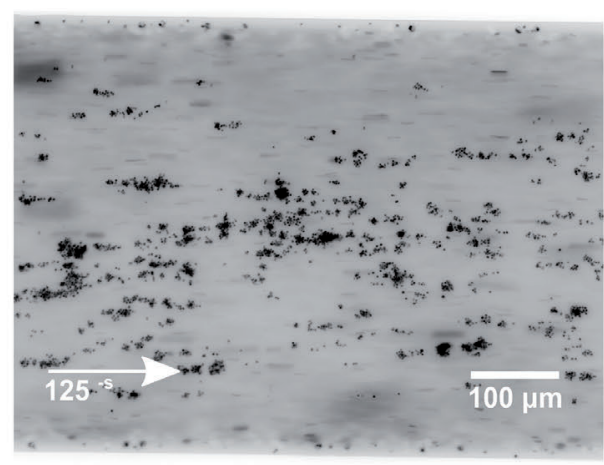

B i

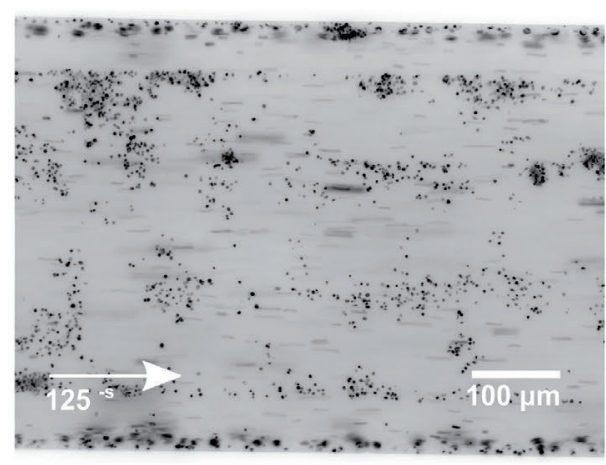

C

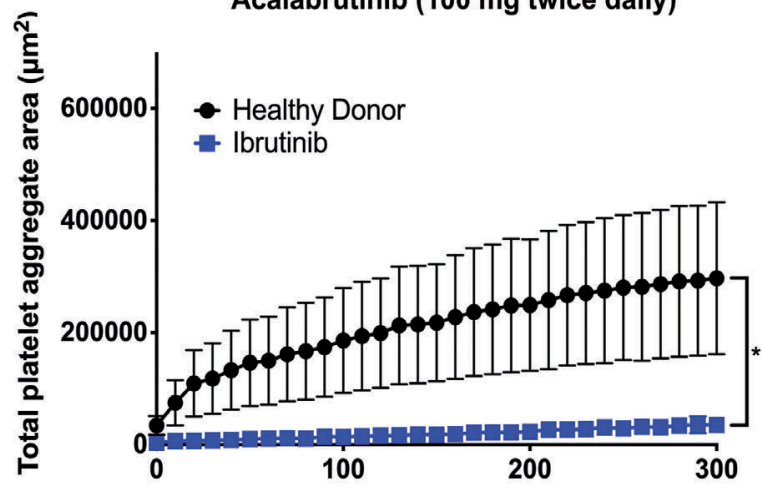

A ii

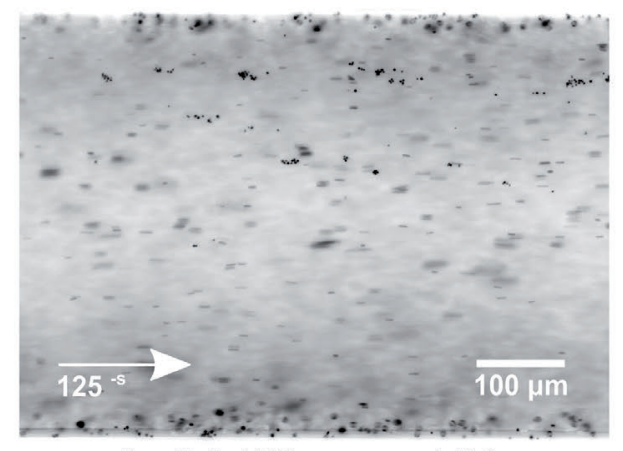

B ii

D

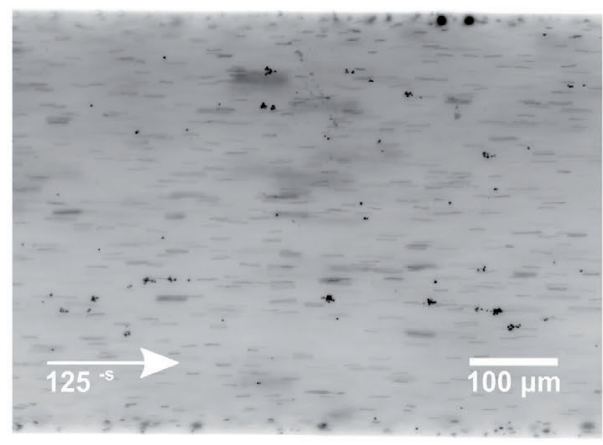

XLA

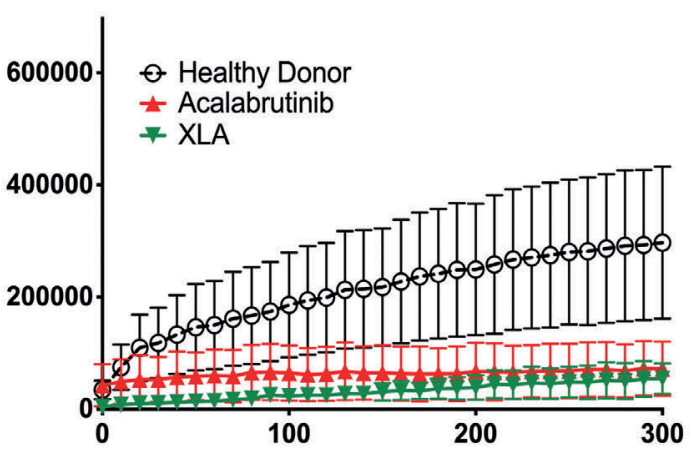

F

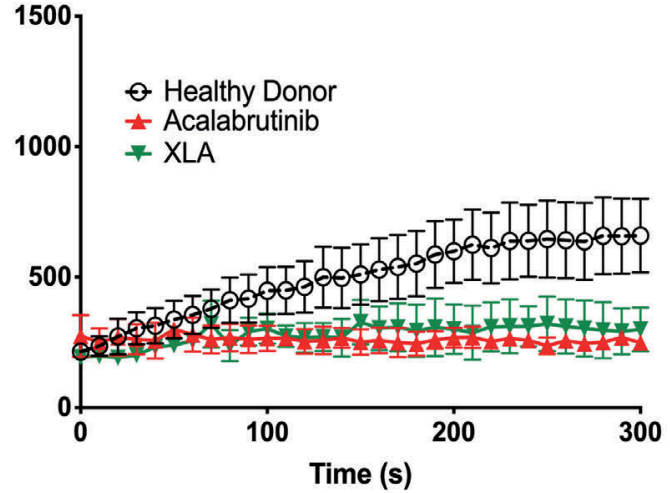

Figure 2. Btk inhibition blocks platelet adhesion to podoplanin under venous flow conditions. (A, B) Whole blood from healthy donors, patients treated with ibrutinib or acalabrutinib or those with X-linked agammaglobulinemia (XLA) was incubated with DiOC dye for 5 min before being flowed across a capillary coated with podoplanin-Fc $(100 \mu \mathrm{g} / \mathrm{mL})$ at $125 \mathrm{~s}^{-1}$ for $5 \mathrm{~min}$. Images were taken every second using fluorescent channels on a Zeiss Axio inverted microscope at $20 \mathrm{X}$ magnification. Representative images from healthy donors (Ai), patients treated with ibrutinib $420 \mathrm{mg}$ once daily (Aii), patients treated with acalabrutinib $100 \mathrm{mg}$ twice daily (Bi) or patients with XLA (Bii). Ilastik 1.1.2 machine learning software was used to automatically and reproducibly identify platelets. Data on platelet surface area coverage and cluster size were measured using the KNIME 3.4 analytics platform. (C, D) Mean \pm standard error of mean (SEM) showing the increases in total platelet aggregate area over time of healthy donors $(n=4)$ and ibrutinib-treated patients $(n=5)(C)$ and in alabrutinib-treated patients $(n=2)$ and patients with XLA $(n=2)(D)$. $(E, F)$ Mean \pm SEM showing increases in mean aggregate size over time of healthy donors and ibrutinib-treated patients (E) and of acalabrutinib-treated patients and patients with XLA, with the curve from healthy donor platelets included for comparison (F). The statistical analysis was performed with two-way analysis of variance. $\star P<0.05$, ns $=$ not significant. 
A i
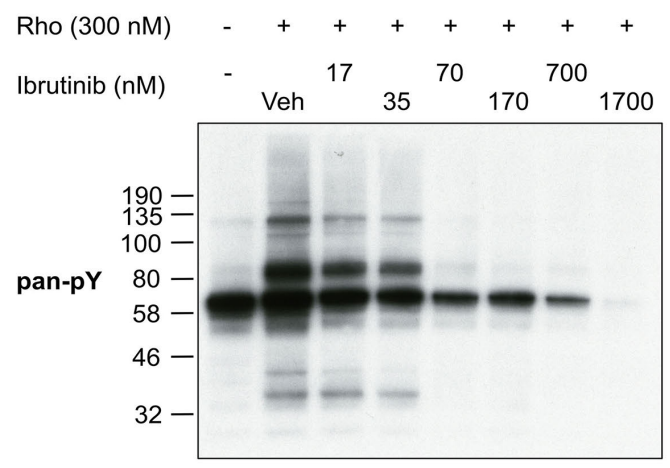

Src pY418

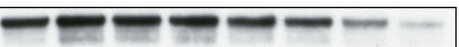

Syk pY525/6

$-1-$

SLP-76 pY145

LAT pY200

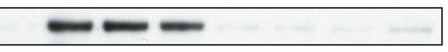

Btk pY551

Btk pY223

PLCy2 pY1217

Total Syk

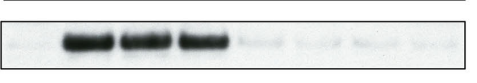

B i

$\operatorname{ADP}(10 \mu \mathrm{M})$

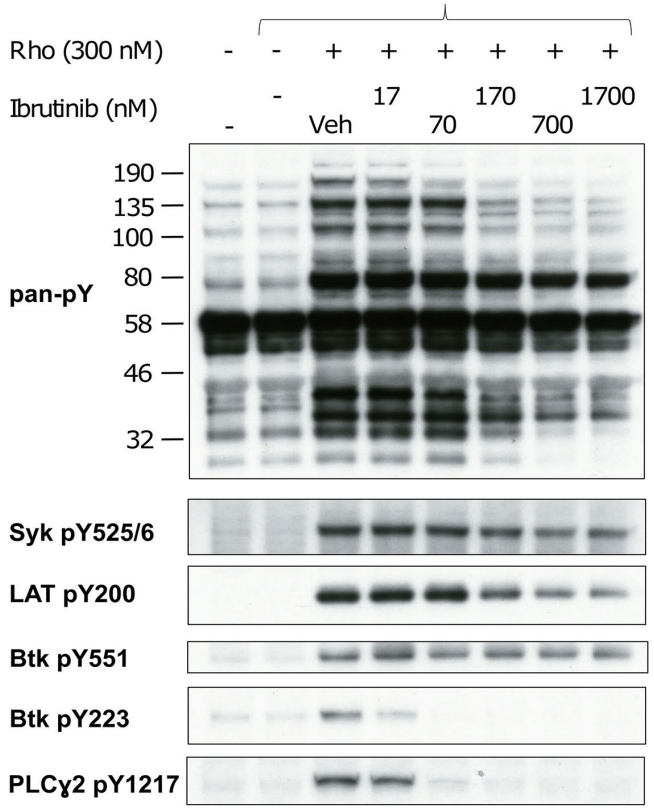

Total Syk

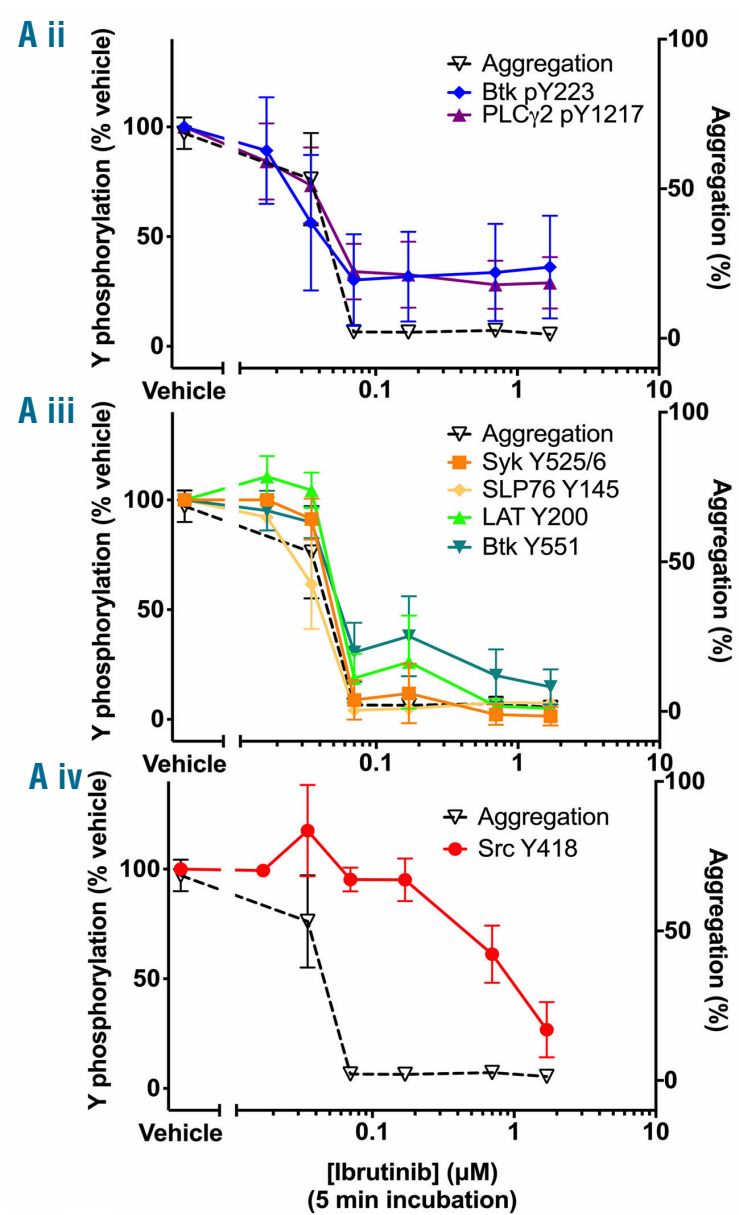

B ii

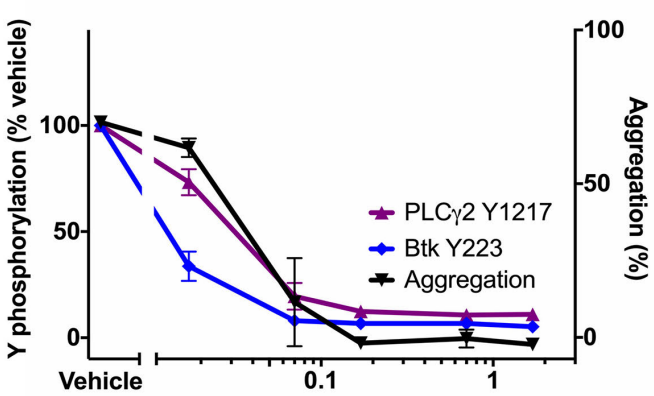

B iii

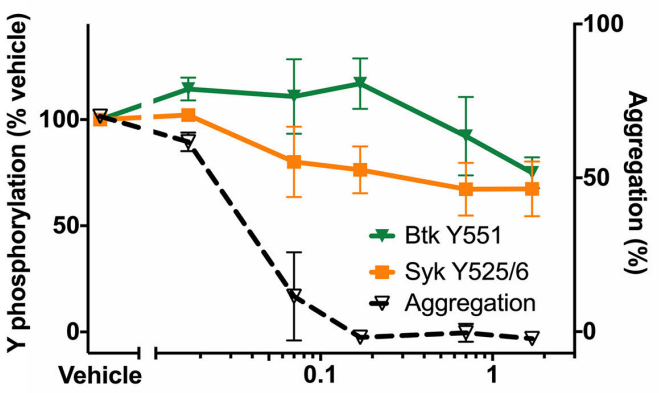

[lbrutinib] $(\mu \mathrm{M})$

$(5 \mathrm{~min}$ incubation)

Figure 3. Low concentrations of ibrutinib block all phosphorylation events downstream of platelet CLEC-2 ligation. Blockade upstream of Btk pY223 is rescued by addition of excess ADP. (A, B) Healthy donor washed human platelets at $4 \times 10^{8} / \mathrm{mL}$ were incubated in the presence of eptifibatide $9 u \mathrm{M}$ with ibrutinib or vehicle for 5 min without (A) or with (B) ADP at $10 \mu \mathrm{M}$ prior to stimulation with $300 \mathrm{nM}$ rhodocytin. Platelets were then lysed with $5 \mathrm{X}$ reducing sample buffer 5 min after addition of agonist. Whole cell lysates were then separated by sodium dodecylsulfate polyacrylamide gel electrophoresis and western blots were probed for whole cell phosphorylation or kinase phosphorylation with the stated antibodies downstream of the platelet CLEC-2 receptor. A representative blot from four identical experiments (i). Mean tyrosine phosphorylation levels \pm standard error of mean (SEM) of four identical experiments for phosphorylation events downstream (ii) and upstream (iii) of Btk pY223 as well as Src pY418 (iv). The mean aggregation trace from Figure $1 \mathrm{~B}$ is included as a dotted line to aid comparison. Mean $\pm \mathrm{SEM}$ of three identical experiments for light transmission aggregometry measurements in the presence of ADP 5 min after stimulation with $300 \mathrm{nM}$ rhodocytin are shown as a solid line in (ii) and as a dotted line in (iii). Rho: rhodocytin. 
A i

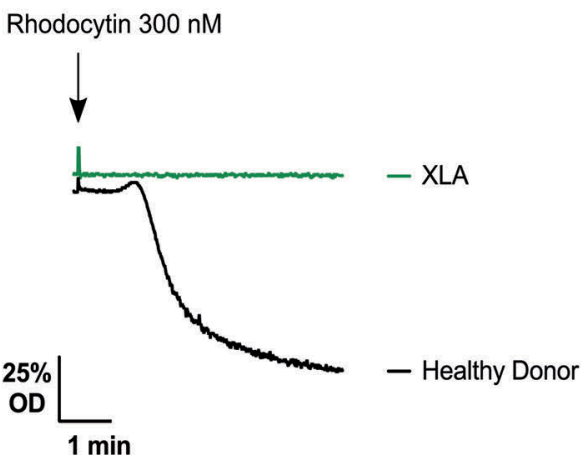

A ii

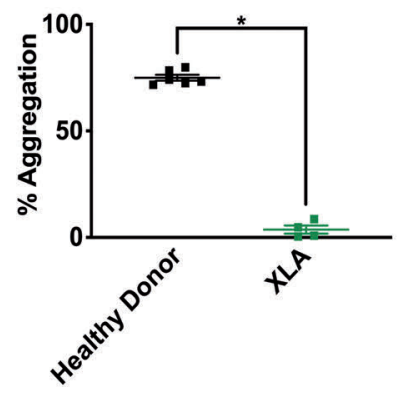

B i
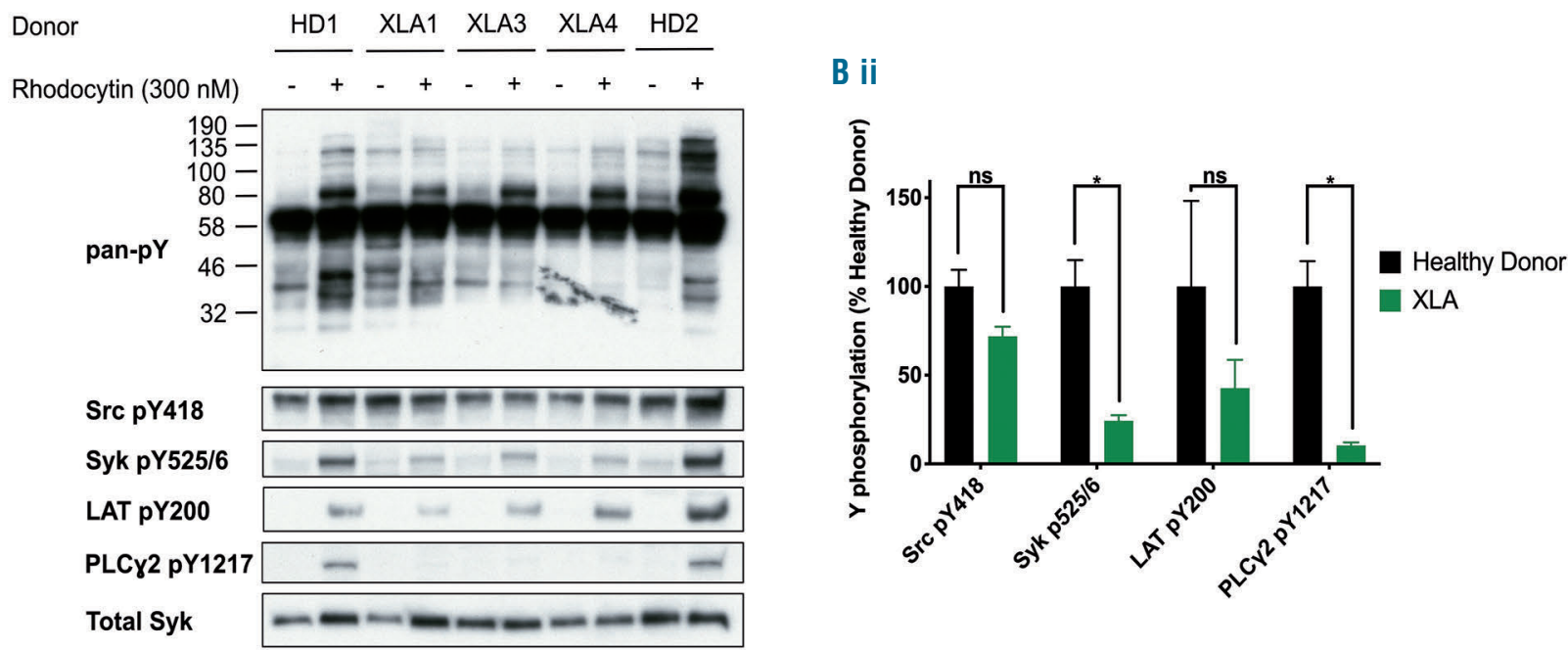

Figure 4. Platelets from patients with X-linked agammaglobulinemia do not aggregate in response to ligation of CLEC-2, but signaling events upstream of Btk are partially preserved. (A) Platelet-rich plasma was isolated from the blood of patients with X-linked agammaglobulinemia (XLA) and stimulated with rhodocytin. (i) Representative trace and (ii) mean \pm standard error of mean (SEM) (healthy donors, $n=6$; XLA patients, $n=4$ ) of optical density 5 min after stimulation with rhodocytin $300 \mathrm{nM}$. Statistical analysis was performed with a two-tailed $t$-test. (B) Eptifibatide $(9 \mu \mathrm{M})$-treated washed platelets at $4 \times 10^{8} / \mathrm{mL}$ from patients with XLA were lysed with $5 \mathrm{X}$ reducing sample buffer $5 \mathrm{~min}$ after addition of rhodocytin $300 \mathrm{nM}$ or phosphate-buffered saline as shown. Whole cell lysates were then separated by sodium dodecylsulfate polyacrylamide gel electrophoresis and western blots were probed for whole cell or tyrosine phosphorylation with the stated antibodies. (i) Blot and (ii) mean \pm SEM $(n=3)$ for phosphorylation events downstream of the CLEC-2 receptor. Results were analyzed statistically using a one-way analysis of variance with Sidak correction for multiple comparisons. OD: optical density.

\section{The kinase domain of Btk is required for signaling} downstream of CLEC-2

We have previously shown that Btk supports platelet activation by GPVI through acting both as a kinase and as an adapter protein. ${ }^{16}$ However, the observation of complete loss of aggregation in the presence of ibrutinib and acalabrutinib suggests that the kinase activity of Btk is critical for activation by CLEC-2. Consistent with this, wild-type but not kinase-dead Btk restored NFAT signaling in a CLEC2-transfected cell line model, which was blocked by ibrutinib and acalabrutinib (Figure 5A-C). CLEC-2 and Btk expression was similar regardless of the Btk construct used (Figure 5D, E). The lack of signaling with kinase-dead Btk confirms a fundamental difference between the role of Btk in the CLEC-2 and GPVI signaling pathways.

\section{Btk does not lie upstream of Syk in CLEC-2-stimulated mouse platelets}

We extended the studies to investigate the role of Btk in CLEC-2 signaling in mouse platelets. In agreement with the results of Lee et al. ${ }^{28}$ we found that only high concentrations of ibrutinib, which abrogated platelet activation by
GPVI, blocked CLEC-2-mediated platelet aggregation (Figure 6A, Table 1 and data not shown). Phosphorylation of Syk Y519/520 (equivalent to Y525/526 in human platelets), and LAT Y132 and Y200 was preserved in the presence of ibrutinib (Figure 6B). This is consistent with the results in human platelets stimulated by CLEC- 2 in the presence of $\mathrm{ADP}$ and further confirms that Btk does not lie upstream of Syk and LAT in mouse platelets.

\section{The effect of ibrutinib on deep vein thrombosis}

We sought to establish whether ibrutinib would prevent IVC thrombus formation in a mouse model of deep vein thrombosis (DVT) that is known to be dependent on CLEC-2. Mice were dosed with ibrutinib via intraperitoneal injection for up to 4 days. This dosing strategy blocked CLEC-2-mediated but not PAR4-mediated platelet activation (Figure 7A). Mice dosed with ibrutinib had a reduction in thrombus prevalence relative to controls (Figure 7Bi) but this was not statistically significant. In the small number of ibrutinib-treated mice which still formed IVC thrombi, thrombus size was not significantly altered relative to that in controls (Figure $7 \mathrm{Bii}$ ). 
A

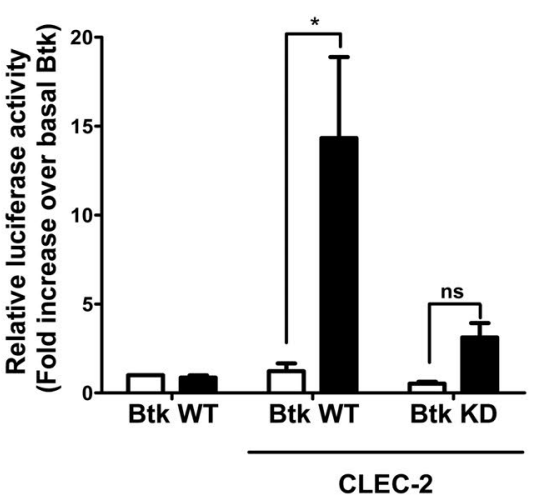

C

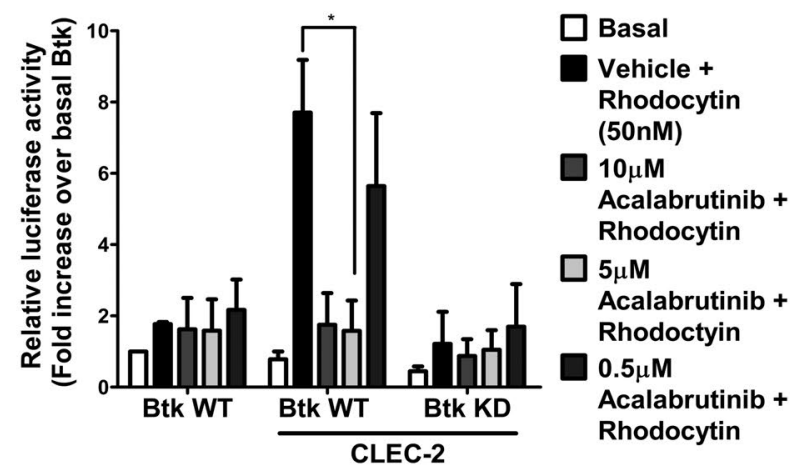

E

WB: Btk

Basal

Rhodocytin

B

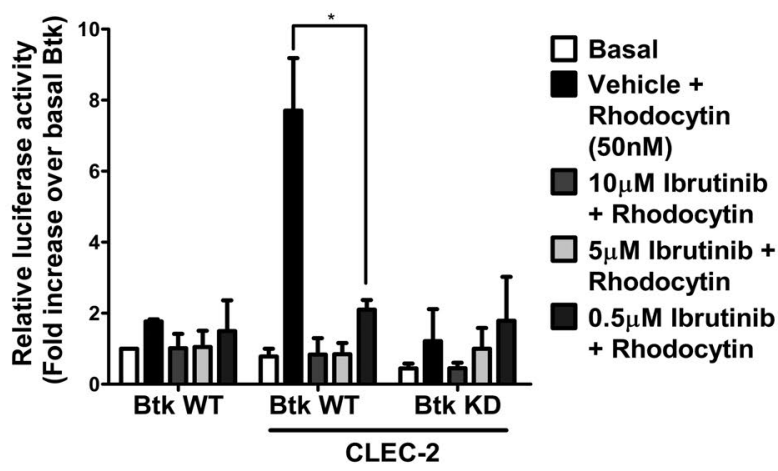

D

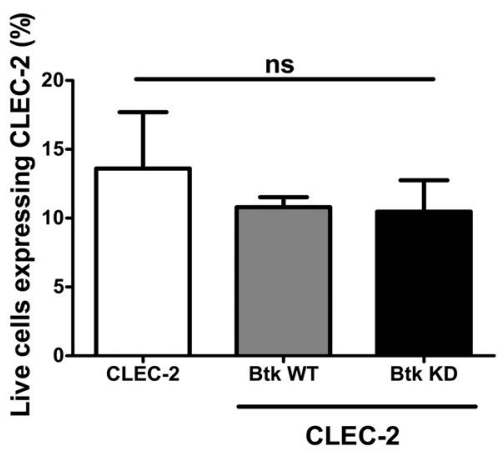

CLEC-2

Btk WT

Btk KD

WB: Tubulin $=0$

Figure 5. NFAT luciferase activity in Btk deficient DT40 cells is restored with transfection of wild-type but not kinase-dead Btk. Btk-deficient DT40 cells were transfected with either wild-type or kinase-dead Btk with or without CLEC-2. All cells were transfected with a NFAT-luciferase reporter plasmid. Cells were stimulated with rhodocytin $300 \mathrm{nM}$ in the presence of serum. (A) Luciferase activity was measured and is shown as mean \pm standard error of mean (SEM) of five identical experiments. (B, C) Cells were stimulated with rhodocytin $50 \mathrm{nM}$ in the presence or absence of ibrutinib (0.5 - $10 \mu \mathrm{M})(\mathrm{B})$ and acalabrutinib (0.5 - $10 \mu \mathrm{M})(\mathrm{C})$. Serum was excluded during stimulation to avoid plasma binding of the drugs. Luciferase activity of vehicle- and drug-treated samples was measured and the difference is shown as the mean \pm SEM of three independent experiments. (D) CLEC-2 expression in the different transfection conditions. (E) Representative western blot showing Btk expression levels in cells transfected with different Btk constructs $(n=5) .{ }^{*} P<0.05$, ns $=$ not significant. WT: wild-type; KD: kinase-dead; WB: western blot.

\section{Discussion}

The main findings from the above studies into the role of Btk downstream of CLEC-2 receptor ligation in platelets are: (i) Btk lies downstream of Syk in the CLEC2 signaling cascade in human and mouse platelets, in contrast to the conclusion of Manne et al.; and (ii) Btk inhibitors selectively block platelet activation by CLEC-2 relatively to GPVI. This suggests that Btk inhibitors can be used to selectively block CLEC-2-mediated platelet activation in thrombo-inflammation without any increase in bleeding or other side effects due to blockade of GPVI and ITAM receptors in other cells.

The results described here do not support the conclusions of Manne et al. that Btk lies upstream of Syk in CLEC-2 signaling in both human and mouse platelets. ${ }^{26}$ While we were able to replicate the complete loss of whole cell tyrosine, Syk and Btk phosphorylation with ibrutinib in human platelets reported by Manne et al., we show that ibrutinib blocks Btk phosphorylation at its Src phosphorylation site (Y551) which is not in keeping with the known mechanism of action of ibrutinib: Btk is phosphorylated by Src at Y551 but ibrutinib then blocks subsequent autophosphorylation at Y223. We also report loss of Btk phosphorylation using the Syk inhibitor PRT 060318. Both of these findings are inconsistent with a model in which Btk lies upstream of Syk. This is further supported by the observation that phosphorylation of Syk and Btk Y551 is restored in the presence of the secondary messenger ADP. These results demonstrate that the phosphorylation of Syk is initially independent of Btk and is then increased by a pathway that involves Btk and ADP.

Our results show that human CLEC-2-mediated platelet activation is critically dependent on the kinase function of Btk. Platelets exposed to low concentrations of ibrutinib and the more Btk-selective acalabrutinib, as well as platelets from patients treated with these inhibitors and patients with genetic loss-of-function mutations of Btk, do 


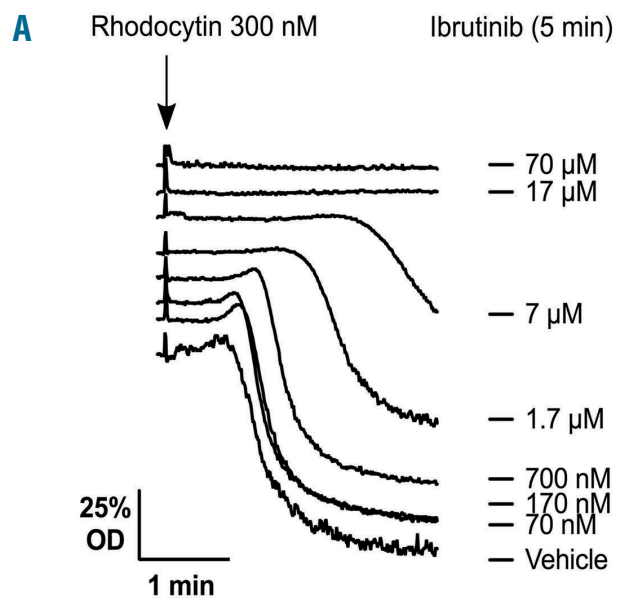

Mouse platelets

B i

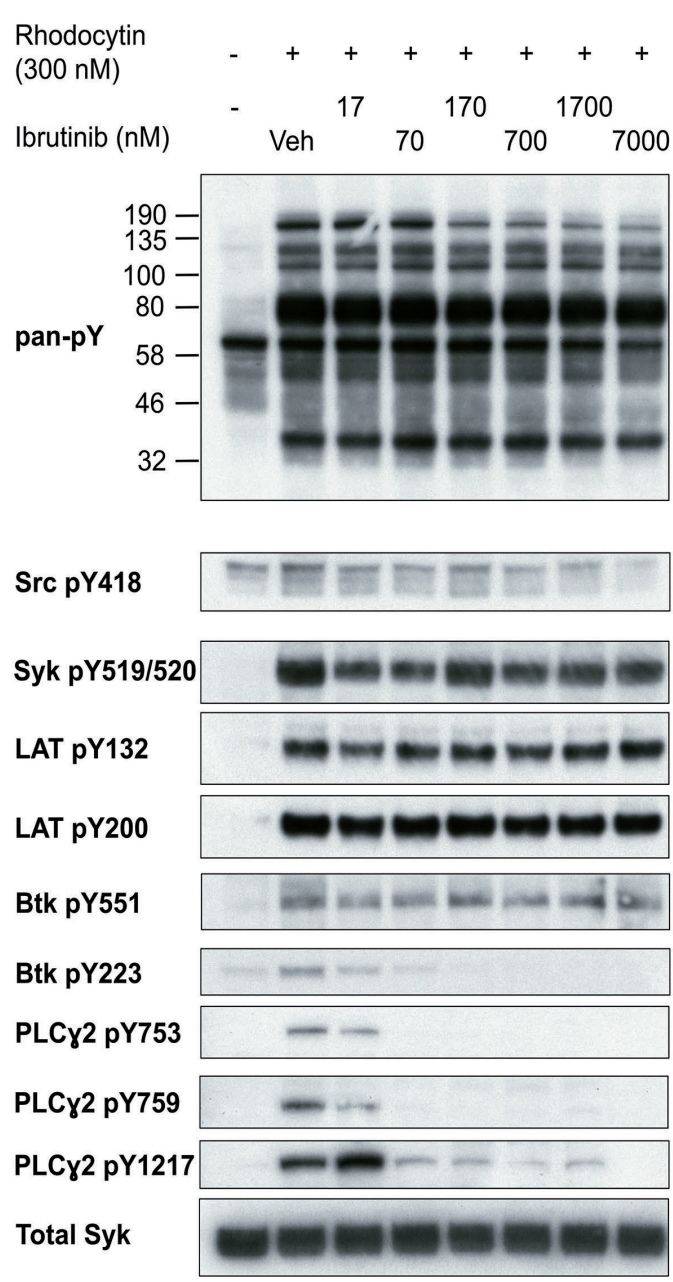

B ii

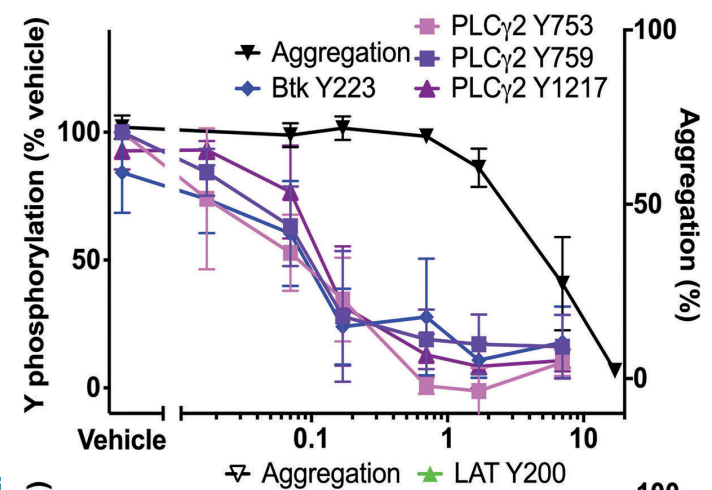

B iii

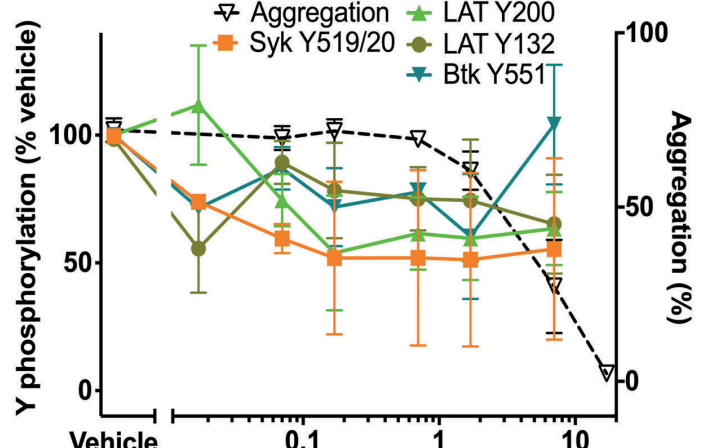

B iv

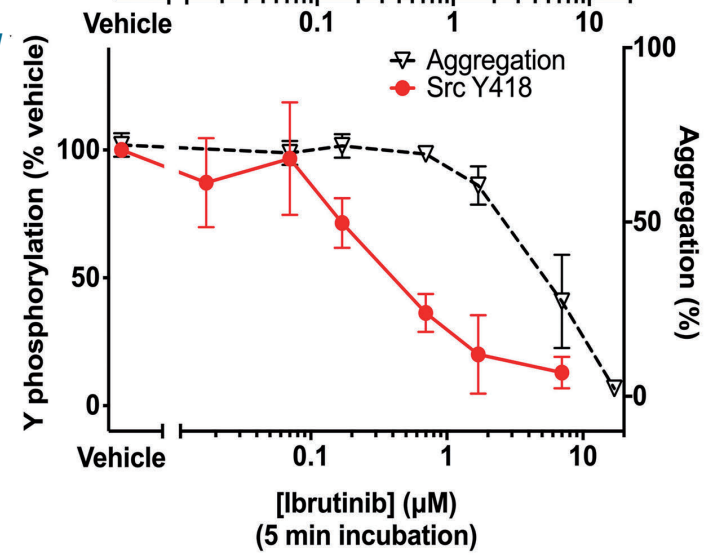

Figure 6. Ibrutinib blocks CLEC-2-mediated tyrosine phosphorylation of Btk pY223 at low concentrations in mouse platelets. Aggregation is only blocked at $\sim 250$-fold higher concentrations. (A) Wild-type mouse washed platelets at $4 \times 10^{8} / \mathrm{mL}$ were incubated with vehicle or ibrutinib for 5 min prior to stimulation with rhodocytin $300 \mathrm{nM}$ for $5 \mathrm{~min}$. A representative light transmission aggregometry trace from three identical experiments is shown. (B) Eptifibatide ( $9 \mu \mathrm{M})$-treated wild-type mouse washed platelets at $4 \times 10^{8} / \mathrm{mL}$ were incubated with ibrutinib or vehicle for 5 min before being stimulated with rhodocytin $300 \mathrm{nM}$. Platelets were then lysed with $5 \mathrm{X}$ reducing sample buffer $5 \mathrm{~min}$ after addition of an agonist. Whole cell lysates were then separated by sodium dodecylsulfate polyacrylamide gel electrophoresis and western blots were probed for whole cell phosphorylation or kinase phosphorylation with the stated antibodies downstream of the platelet CLEC-2 receptor. (i) Representative blots and (iiiv) mean results of three identical experiments examining degree of aggregation and tyrosine phosphorylation levels of the proteins shown. Mean aggregation ( $n=3$ ) results are shown in (ii) and are included as dotted lines in (iii-iv) to aid comparison. All results are shown as mean \pm standard error of mean. OD: optical density. 
Ai
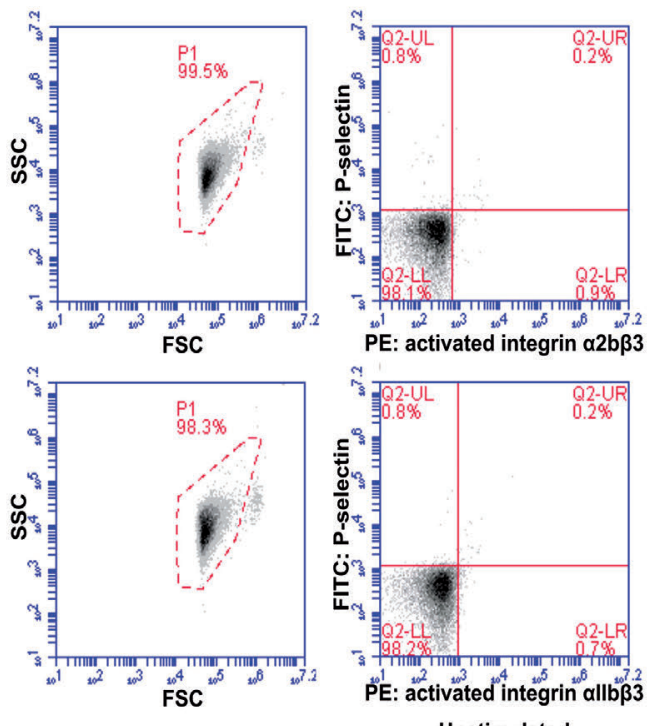

Unstimulated
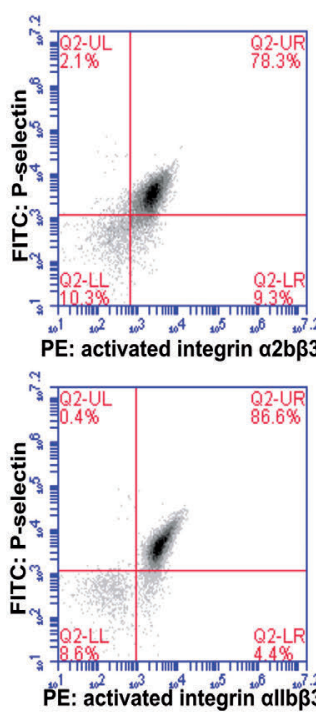

PAR4 $500 \mu \mathrm{M}$

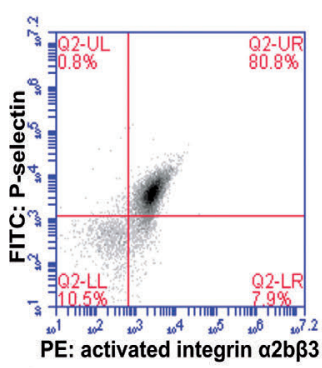

2 hours after pre-surgical dose of vehicle

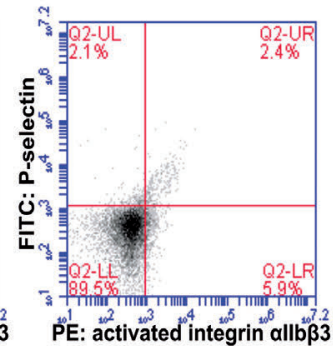

Rhodocytin $300 \mathrm{nM}$

A ii

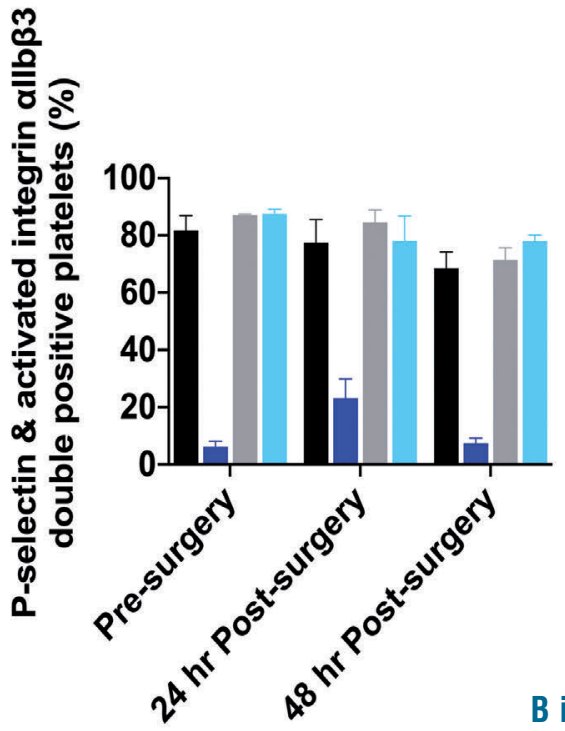

Rhodocytin $300 \mathrm{nM}($ Veh)

Rhodocytin $300 \mathrm{nM}$ (lbr)

PAR4 $500 \mathrm{nM}$ (Veh)

PAR4 $500 \mathrm{nM}$ (lbr)

B i

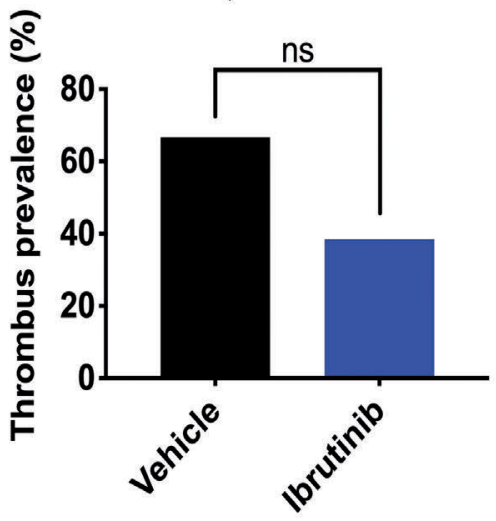

B ii

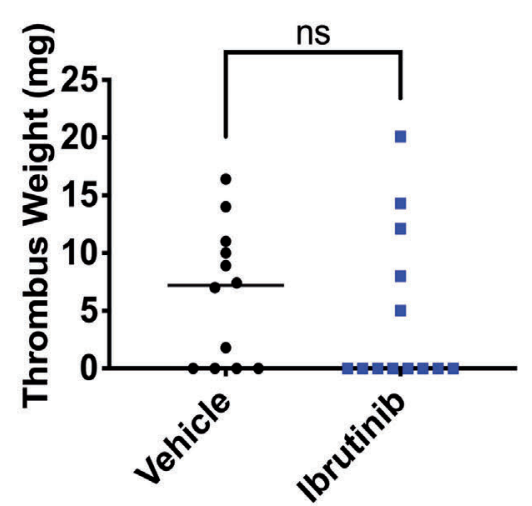

Figure 7. Mice dosed with ibrutinib have a trend towards reduction of thrombus formation in an in vivo venous thrombosis model. Wild-type mice were dosed with ibrutinib (35- $70 \mathrm{mg} / \mathrm{kg}$ ) or vehicle for 1 - 3 days before and 2 days following inferior vena cava (IVC) stenosis surgery to achieve consistent CLEC-2 inhibition throughout the post-surgical period. (A) Heparinized whole blood was collected via tail bleeding from mice undergoing the same dosing schedule but not undergoing surgery at the stated time points. The blood was incubated with FITC-conjugated anti-P-selectin and PE-conjugated anti-activated integrin $\alpha$ llb $\beta 3$ antibodies for 30 min before undergoing red cell lysis and fixation and analysis using flow cytometry. The effect on platelet activation after stimulation with PAR4 peptide (500 nM) and rhodocytin $(300 \mathrm{nM})$ is shown as (i) representative plots, (ii) mean data \pm standard error of mean (ibrutinib, $n=6$; vehicle, $n=3$ ). (B) Two hours following the pre-surgical dose of ibrutinib or vehicle, IVC stenosis was induced under general anesthesia with a ligature. Mice were allowed to recover and were then culled $48 \mathrm{~h}$ later and examined for the size of any IVC thrombus. (i) Thrombus prevalence and (ii) thrombus weight are shown (ibrutinib, $n=13$; vehicle, $n=12$ ). The horizontal line represents the median thrombus weight. A Fisher exact test was used for the statistical analysis of thrombus prevalence, whereas a Mann-Whitney test was used for the analysis of weight. ns=not significant. SSC: side scatter; FSC: forward scatter. 
not demonstrate any CLEC-2-mediated activation, even when stimulated with very high levels of agonist. We have previously shown that genetic or pharmacological Btk inhibition of platelets does not result in loss of activation in response to GPVI in human platelets because: (i) Btk was able to function as an adapter downstream of GPVI to preserve platelet activation; and (ii) Tec may be able to compensate for the lack of Btk kinase or adapter activity. ${ }^{16}$ Here we show that Tec is not able to compensate for a lack of Btk in the CLEC-2 signaling cascade through studies on patients with XLA and that the kinase domain of Btk is critical for CLEC-2 signaling in a transfected cell line model.

The present results suggest that Btk inhibitors have the potential to be used at very low concentrations as selective inhibitors of CLEC-2 in thrombo-inflammatory disorders with minimal off-target effects and no increased bleeding. Not only does Btk inhibition prevent platelet activation in response to CLEC-2 but we have shown that it also results in abrogation of platelet adhesion to podoplanin at venous rates of shear and a reduction in DVT formation in ibrutinib-treated mice. The in vivo DVT study was powered based on the rates of thrombosis seen in CLEC-2-deficient and WT mice in the studies by Payne et al. ${ }^{3}$ The lack of statistical significance in the trend towards inhibition of DVT formation may reflect the fact that pharmacological blockade of Btk in mice did not result in full inhibition of CLEC-2 signaling throughout the $48 \mathrm{~h}$ required for venous thrombosis in this model and the fact that, unlike for human CLEC-2 signaling, mouse CLEC-2 is not critically dependent on Btk. Interestingly the thrombi that did form in the ibrutinibtreated mice were the same size as those in the control mice. This is consistent with a model in which CLEC-2 activation is required to initiate formation of a thrombus, but not its propagation.

In conclusion, the present study shows that Btk lies downstream of Syk in human and mouse platelets and that Btk inhibitors block human CLEC-2-mediated platelet function at concentrations more than 20 -fold lower than those that block GPVI signaling ${ }^{16}$ and mouse CLEC-2. The mechanism underlying this selectivity is that Btk kinase activity is critical for human platelet CLEC-2 function, but not for GPVI. This means that low- dose Btk inhibitors could be used therapeutically to inhibit CLEC-2 in platelets without any off-target effects on GPVI and ITAM receptors in other hematopoietic cells. This provides a mechanism for selective targeting of CLEC-2 in thrombo-inflammatory disorders, such as DVT or infection-driven thrombosis, while preserving hemostasis. ${ }^{3,4}$

\section{Disclosures}

This work was supported by British Heart Foundation (BHF) Programme grant (RG/13/18/30563), a BHF Clinical Fellowship to PLRN (FS/17/20/32738), a BHF Senior Basic Science Research Fellowship to AB (FS/19/30/34173), an AMS springboard grant to AYP (SBF002\1099) and the University of Birmingham's Institute of Translation Medicine and Institute of Cardiovascular Sciences; SPW holds a BHF Chair (CH03/003). JAE is supported by the Deutsche Forschungsgemeinschaft (DFG grant: Eb177/13-1). Btk deficient DT40 cells, plasmid constructs and rabbit anti-Btk antibody were a kind gift from Dr Mike Tomlinson (University of Birmingham, UK). PLR Nicolson has received research grants from Rigel Pharmaceuticals, Novartis Pharmaceuticals and materials from Principia Biopharma.

\section{Contributions}

PLRN, CEH, SHN and SPW conceived the study and wrote the manuscript. PLRN, SHN, JH, LG-Q, CWS, JC, AB, AOK, NSP, DNK, SW and CNW performed experiments. PLRN, $S H N, J H$ and JAP performed data analysis. HC and APH consented and provided access to patients with XLA. JAE provided key reagents. GP consented and provided access to patients with CLL. CEH, AYP and SPW supervised the study. All authors critically appraised the manuscript.

\section{Acknowledgments}

This work was supported by a British Heart Foundation (BHF) Programme grant (RG/13/18/30563), a BHF Clinical Fellowship to PLRN (FS/17/20/32738), a BHF Senior Basic Science Research Fellowship to AB (FS/19/30/34173), an AMS springboard grant to AYP (SBF002\1099) and the University of Birmingham's Institute of Translation Medicine and Institute of Cardiovascular Sciences; SPW holds a BHF Chair (CH03/O03). JAE is supported by the Deutsche Forschungsgemeinschaft (DFG grant: Eb177/13-1).

\section{References}

1. Lowe K, Watson SP, Lax S, Frampton J. CLEC-2 is not required to maintain separation of intact blood and lymphatic vasculature. Blood. 2015;123(20):3200-3207.

2. Astarita JL, Acton SE, Turley SJ. Podoplanin: emerging functions in development, the immune system, and cancer. Front Immunol. 2012;3(283):1-11.

3. Payne H, Ponomaryov T, Watson SP, Brill A. Mice with a deficiency in CLEC-2 are protected against deep vein thrombosis. Blood. 2017;129(14):2013-2020.

4. Hitchcock JR, Cook CN, Bobat S, et al. Inflammation drives thrombosis after Salmonella infection via CLEC- 2 on platelets. J Clin Invest. 2015;125(12):4429-4446.

5. Rayes J, Watson SP, Nieswandt B. Functional significance of the platelet immune receptors GPVI and CLEC-2. J Clin Invest.
2019;129(1):12-23.

6. Severin S, Pollitt AY, Navarro-Núñez L, et al. Syk-dependent phosphorylation of CLEC-2: a novel mechanism of hem-immunoreceptor tyrosine-based activation motif signaling. J Biol Chem. 201;286(6):4107-4116.

7. Spalton JC, Mori J, Pollitt AY, Hughes CE, Eble JA, Watson SP. The novel Syk inhibitor $\mathrm{R} 406$ reveals mechanistic differences in the initiation of GPVI and CLEC-2 signaling in platelets. J Thromb Haemost. 2009;7(7): 1192-1199.

8. Pollitt AY, Grygielska B, Leblond B, Desire L, Eble JA, Watson SP. Phosphorylation of CLEC-2 is dependent on lipid rafts, actin polymerization, secondary mediators, and Rac. Blood. 2010;115(14):2938-2946.

9. Hughes CE, Finney BA, Koentgen F, Lowe KL, Watson SP. The N-terminal SH2 domain of Syk is required for (hem)ITAM, but not integrin, signaling in mouse platelets. Blood. 2015;125(1):144-154.
10. Fuller G, Williams J, Tomlinson MG, et al. The C-type lectin receptors CLEC-2 and Dectin-1, but not DC-SIGN, signal via a novel YXXL-dependent signaling cascade. J Biol Chem. 2007;282(17):12397-12409.

11. Hughes CE, Pollitt AY, Mori J, et al. CLEC-2 activates Syk through dimerization. Blood. 2010;115(14):2947-2955.

12. Borgognone A, Navarro-Núñez L, Correia JN, et al. CLEC-2-dependent activation of mouse platelets is weakly inhibited by cAMP but not by cGMP. J Thromb Haemost. 2014;12(4):550-559.

13. Byrd JC, Furman RR, Coutre SE, et al. Targeting BTK with ibrutinib in relapsed chronic lymphocytic leukemia. N Engl J Med. 2013;369(1):32-42.

14. Byrd JC, Harrington B, O'Brien S, et al. Acalabrutinib (ACP-196) in relapsed chronic lymphocytic leukemia. N Engl J Med. 2016; 374(4):323-332.

15. Wang M, Rule S, Zinzani PL, et al 
Acalabrutinib in relapsed or refractory mantle cell lymphoma (ACE-LY-004): a singlearm, multicentre, phase 2 trial. Lancet. 2018;391(10121):659-667.

16. Nicolson PLR, Hughes CE, Watson S, et al. Inhibition of Btk by Btk-specific concentrations of ibrutinib and acalabrutinib delays but does not block platelet aggregation to GPVI. Haematologica. 2018;103(12):2097-2108.

17. Burkhart JM, Vaudel M, Gambaryan S, et al. The first comprehensive and quantitative analysis of human platelet protein composition allows the comparative analysis of structural and functional pathways. Blood. 2012;120(15):e73-82.

18. Zeiler M, Moser M, Mann M. Copy number analysis of the murine platelet proteome spanning the complete abundance range. Mol Cell Proteomics. 2014;13(12):3435-3445

19. Quek LS, Bolen J, Watson SP. A role for Bruton's tyrosine kinase (Btk) in platelet activation by collagen. Curr Biol. 1998;8(20): 1137-1140.

20. Atkinson BT, Ellmeier W, Watson SP. Tec regulates platelet activation by GPVI in the absence of Btk. Blood. 2003;102(10):35923599.

21. Busygina K, Denzinger V, Bernlochner I, Weber C, Lorenz R, Siess W. Btk inhibitors as first oral atherothrombosis-selective antiplatelet drugs? Thromb Haemost. 2019;119(08):1212-1221.

22. Levade M, David E, Garcia C, et al. Ibrutinib treatment affects collagen and von Willebrand factor-dependent platelet functions. Blood. 2014:124(26):3991-3995.

23. Kamel S, Horton L, Ysebaert L, et al. Ibrutinib inhibits collagen-mediated but not ADP-mediated platelet aggregation. Leukemia. 2015;29(4):783-787.

24. Bye AP, Unsworth AJ, Desborough MJ, et al. Severe platelet dysfunction in NHL patients receiving ibrutinib is absent in patients receiving acalabrutinib. Blood Adv. 2017;1(26):2610-2623.

25. Busygina K, Jamasbi J, Seiler T, et al. Oral Bruton tyrosine kinase inhibitors selectively block atherosclerotic plaque-triggered thrombus formation in humans. Blood. 2018;131(24):2605-2616.

26. Manne BK, Badolia R, Dangelmaier C, et al. Distinct pathways regulate syk protein activation downstream of immune tyrosine activation motif (ITAM) and hemITAM receptors in platelets. J Biol Chem. 2015;290(18):11557-11568.

27. Koessler J, Hermann S, Wener K, et al. Role of purinergic receptor expression and function for reduced responsiveness to adenosine diphosphate in washed human platelets. PLoS One. 2016; 11(1):e0147370

28. Lee RH, Piatt R, Conley PB, Bergmeier W Effects of ibrutinib treatment on murine platelet function during inflammation and in primary hemostasis. Haematologica. 2017;102(3):e89-e92.

29. Gitz E, Pollitt AY, Gitz-Francois JJ, et al. CLEC-2 expression is maintained on activated platelets and on platelet microparticles. Blood. 2014;124(14):2262-2270.

30. Suzuki-Inoue K, Fuller G, Garcia A, et al. A novel Syk-dependent mechanism of platelet activation by the C-type lectin receptor CLEC-2. Blood. 2006;107(2):542-549.

31. Navarro-Núñez L, Pollitt AY, Lowe K, Latif A, Nash GB, Watson SP. Platelet adhesion to podoplanin under flow is mediated by the receptor CLEC-2 and stabilised by Src/Sykdependent platelet signalling. Thromb Haemost. 2015;113(5):1109-1120.

32. Fillbrunn A, Dietz C, Pfeuffer J, Rahn R, Landrum GA, Berthold MR. KNIME for reproducible cross-domain analysis of life science data. J Biotechnol. 2017;261:149156.

33. Sommer C, Straehle C, Kothe U, Hamprecht FA. Ilastik: interactive learning and segmentation toolkit. 2011 IEEE International Symposium on Biomedical Imaging: From Nano to Macro. IEEE. 2011:230-233. 